\title{
Green technologies use in the smart grid construction in rural power supply systems
}

\author{
Igor Naumov ${ }^{1,2}$, Sergey Podyachikh ${ }^{1, *}$, Marina Polkovskaya ${ }^{1}$, and Aleksandr Tretyakov ${ }^{1}$ \\ ${ }^{1}$ Irkutsk State Agrarian University Named After A.A. Ezhevsky, Irkutsk, Russia \\ ${ }^{2}$ Irkutsk National Research Technical University, Irkutsk, Russia
}

\begin{abstract}
The article considers the using intelligent controls possibility in low-voltage rural electric networks to minimize the unbalance modes consequences. The proposed technology includes the digital data transmission compilation on the electrical energy parameters with a new balancing technical means the electrical network operating mode. Digital feedback is provided for changes the balancing device (BD) parameters by the unbalancing power consumption changing level. Based on the developed methods compilation, software for calculating unbalancing modes has been created, which makes it possible to assess the currents and voltages unbalancing effect on the power quality and its additional losses change. The "green" technology proposed version, which increases the economic and the electric energy environmental safety use in the rural electric power industry, contains a new constructive solution for the balancing device implementation. The proposed technology was tested on the measurement data basis in existing electrical networks. Based on the MALAB technologies use, changes studied indicators visualization in the before and after BD integration in the electrical network was carried out and its analysis was makes. Used on the "neural networks" MALAB technology, a preventive assessment of the unbalancing power consumption events development in the investigated operating electrical network is presented, as well as the proposed technology effectiveness assessment was carried out.
\end{abstract}

\section{Introduction}

Ensuring safe and reliable power supply to consumers is an essential requirement for the various economic orientation electric power systems. The intelligent networks introduction into modern power supply systems makes it possible to saturate them with new technical means that, without human intervention, can update these systems structure, increase their manageability. Thanks to its ability to control and regulate energy costs, as well as reduce the electrical equipment and its failures, smart grid technology makes it possible to improve the people's lives quality and thereby move towards a sustainable future. In today's deteriorating environmental situation, the automated and safe intellectual devices use in electrical networks has been called "green technologies". Their distinctive feature is the

\footnotetext{
*Corresponding author: psv78@yandex.ru
} 
digital two-way feedback between the control tool and the processes occurring in the power supply system. Such communication is carried out through online software and services. Modern rural power supply systems are insufficiently equipped with control tools, they can be called "low observability" electric networks. In this regard, such "smart, green technologies" development is very relevant and allows us to implement the basic providing electricity requirements to consumers: power supply reliability, power quality, as well as compliance with economic and environmental safety measures. Let's call such technologies "GSG Technology". It has been stated repeatedly in numerous publications about the existing operating modes rural $0.38 \mathrm{kV}$ electric networks that the objective these modes characteristic is the phase currents and voltages unbalance. At the same time, three-phase system unbalance leads to a significant change in quality indicators characterizing the unbalance level which values are set by Standards [1-3]. At the same time, the currents load unbalance not only reduces the power quality level and its attractiveness as a commodity, but also significantly increases its overspending, creating additional losses. Thus, the energy economic attributes saving and power transmission environmental requirements are violated, since the additional losses allocation creates conditions for the fire's occurrence [4-6].

The power quality indicators characterizing the three-phase system unbalance are the negative and zero sequences voltage unbalance coefficients, determined by the expressions:

$$
K_{2 u}=\frac{U_{2}}{U_{1}} \cdot 100 \% \text { and } K_{0 u}=\frac{U_{0}}{U_{1}} \cdot 100 \%
$$

In addition, the evaluating criterion slow voltage changes due to the currents unbalance load in the phases is also the steady-state voltage deviation, determined by the expression:

$$
\delta U=\frac{U-U_{\text {nom. }}}{U_{\text {nom. }}} \cdot 100 \%
$$

In accordance with the Standard [1], this indicator value at the electricity transmission point to the consumer $(0.4 \mathrm{kV}$ buses $)$ should not exceed $\pm 10 \%$. If we are talking about the consumers connection point to the general-purpose electrical network (load node), then in this case, in the $95 \%$ measurement time interval, the indicator value should not exceed \pm $5 \%$.

At the same time, the symmetrical positive, negative and zero sequences currents components cause additional power losses, which can be characterized by a loss coefficient determined by the expression [7]:

$$
K_{P}=1+K_{2 i}^{2}+K_{0 i}^{2} \cdot\left(r_{1}+3 \cdot r_{N}\right)
$$

Where are the coefficients: $K_{2 i}=I_{2} / I_{1}, K_{0 i}=I_{0} / I_{1}$ which are determined based on the positive, negative and zero sequences currents values.

The article purpose is to develop a smart grid technology for assessing and minimizing the unbalancing consequences modes in rural electric networks. To achieve this goal, a tasks number have been formulated:

- based on the developed methods to create an algorithm and software "GSG Technology";

- to consider the using possibility a special balancing device with digital feedback;

- to test the proposed technology to assess the current unbalance mode operation level in the $0.38 \mathrm{kV}$ electrical network;

- to analyze the unbalancing modes and prove the proposed model balancing device effectiveness;

- to carry out the results statistical processing obtained and to prognosis the development unbalancing power consumption events in the existing electrical network. 


\section{Materials and Methods}

With unbalancing power consumption, the positive, negative and zero sequences voltages and currents components can be determined in accordance with the modular calculation method [7]:

$$
\begin{gathered}
U_{1}=\frac{1}{6} \cdot\left\{\left[2 U_{A}+\sqrt{3} \cdot(s+r)-(p+g)\right]+j \cdot[(s-r)+\sqrt{3} \cdot(p-g)]\right\} \\
U_{2}=\frac{1}{6} \cdot\left\{\left[2 \mathrm{U}_{\mathrm{A}}-\sqrt{3} \cdot(\mathrm{s}+\mathrm{r})-(\mathrm{p}+\mathrm{g})\right]+\mathrm{j} \cdot[(\mathrm{s}-\mathrm{r})-\sqrt{3} \cdot(\mathrm{p}-\mathrm{g})]\right\} ; \\
\mathrm{U}_{0}=\frac{1}{3} \cdot\left[U_{A}+(p+g)-j \cdot(s-r)\right] . \\
I_{1}=\left(\frac{1}{6 \cdot I_{A}}\right) \cdot\left\{\left[3 \cdot I_{A}^{2}-I_{A} \cdot(d+\sqrt{3} \cdot g)+2 \cdot \sqrt{3} \cdot g\right]+j\right. \\
\left.\cdot\left[\sqrt{3} \cdot I_{A}^{2}-I_{A} \cdot(\sqrt{3} \cdot d-e)-2 \cdot \sqrt{3} \cdot h\right]\right\} ; \\
I_{2}=\left(\frac{1}{6 \cdot I_{A}}\right) \cdot\left\{\left[3 \cdot I_{A}^{2}-I_{A} \cdot(\sqrt{3} \cdot e-d)-2 \cdot \sqrt{3} \cdot g\right]-j \cdot\left[\sqrt{3} \cdot I_{A}^{2}-I_{A} \cdot(\sqrt{3} \cdot d+e)-2 \cdot \sqrt{3} .\right.\right. \\
h]\} \\
I_{0}=\frac{1}{3} \cdot(d-j \cdot e) .
\end{gathered}
$$

In these expressions: $p=\frac{U_{A}}{2}+\frac{U_{B}^{2}}{2 U_{A}}-\frac{U_{A B}^{2}}{2 U_{A}} ; g=\frac{U_{A}}{2}+\frac{U_{C}^{2}}{2 U_{A}}-\frac{U_{C A}^{2}}{2 U_{A}} ; s=\sqrt{U_{B}^{2}-p^{2}} ; r=$ $\sqrt{U_{C}^{2}-g^{2}} ; g=b_{1} \cdot c_{1}+b_{2} \cdot c_{1} ; h=b_{1} \cdot b_{2}-c_{1} \cdot c_{2} ; \quad c_{1}=\sqrt{I_{A}^{2}-b_{1}^{2}} ; \quad c_{2}=\sqrt{I_{B}^{2}-b_{1}^{2}} ;$ $e=\sqrt{I_{N}^{2}-d}$.

The loss coefficient value (3), which is the energy losses ratio in the unbalancing mode to the corresponding losses caused by the positive sequence currents flow, allows to determine how much energy losses in the real unbalancing mode exceed those losses that can be obtained by taking appropriate measures to minimize the negative and zero sequences currents:

$$
K_{P}=\frac{\Delta W_{U N B .}}{\Delta W_{1}}=\tau \cdot\left(I_{1}^{2} \cdot r_{1}+I_{2}^{2} \cdot r_{2} \cdot I_{0}^{2} \cdot r_{0}\right) / I_{1}^{2} \cdot r_{1} \cdot \tau,
$$

Where $r_{l}, r_{2}, r_{0}$ - active resistances of the positive, negative and zero sequences studied power line, respectively.

Thus, in order to evaluate the electrical network unbalance operation mode, it is necessary to know five currents: $I_{A}, I_{B}, I_{C}, I_{N}, I_{B C}$ and six voltages: $U_{A}, U_{B}, U_{C}, U_{A B}, U_{B C}$, $U_{C A}$.

The $\mathrm{I}_{\mathrm{N}}$ and $\mathrm{I}_{\mathrm{BC}}$ values can be determined based on the corresponding vector diagrams construction as the currents projections geometric sum on the " $\mathrm{X}$ " and "Y" axes:

$$
\begin{aligned}
& I_{N}=\sqrt{\left(I_{n x}\right)^{2}+\left(I_{n y}\right)^{2}} ; \quad I_{n x}=I_{a x}+I_{b x}+I_{c x} ; \quad I_{n y}=I_{a y}+I_{b y}+I_{c y} ; \quad I_{a x}=0 ; \quad I_{a y}=I_{A} ; \\
& I_{b x}=I_{B} * 0.866025 ; \quad I_{b y}=-0.5 * I_{B} ; \quad I_{c x}=-0.866025 * I_{C} ; \quad I_{c y}=-0.5 * I_{C} ; \quad I_{B C}= \\
& \sqrt{\left(I_{B C X}\right)^{2}+\left(I_{B C Y}\right)^{2}} ; I_{B C X}=I_{b x}+I_{c x} ; \quad I_{B C Y}=I_{b y}+I_{c y} .
\end{aligned}
$$

In addition to these values, to evaluate the unbalanced mode, it is necessary to know the high voltage line parameters, supplying power to the $10 / 0.4 \mathrm{kV}$ power transformer - the positive (negative) sequence complex resistance; the power transformer parameters - it's the positive (negative) and zero sequence complex resistance, as well as the $0.38 \mathrm{kV}$ line parameters - the positive (negative) and zero sequence complex resistance. The studied 
indicators determination, characterizing the power quality and its additional losses with special balancing device inclusion into the electrical network is carried out in accordance with the method, described in detail in $[8,9]$.

The conducted studies devoted to the low-voltage electrical networks operating modes [11-18] have established that the most effective balancing means the $038 \mathrm{kV}$ network operating mode is the special balancing devices use with zero-sequence currents minimal resistance. This device type is used mainly in electrical networks that electricity suppliers the utility load consumers (urban and rural).

Balancing devices described in $[19,20]$ can be used as such the BD example.

In this article, we propose to use a balancing device similar in its parameters to the devices $[8,9]$, but differing in that it is a compilation of the digital block and the electrical part block.

The International Electrotechnical Commission (IEC) published the IEC 61850 Standard "Networks and Communication Systems at substations" to solve the constructing problems the digital substations configuration for standardization purposes [21]. In accordance with this Standard, we propose a certain "GSG Technology" type, which functional scheme is shown in Figure 1. Figure 1 shows: 1. Combined optical current and voltage transformer. 2. Computer. 3. Software. 4. A balancing device. 5. The balancing device connection node.

Let's consider the above scheme in more detail.

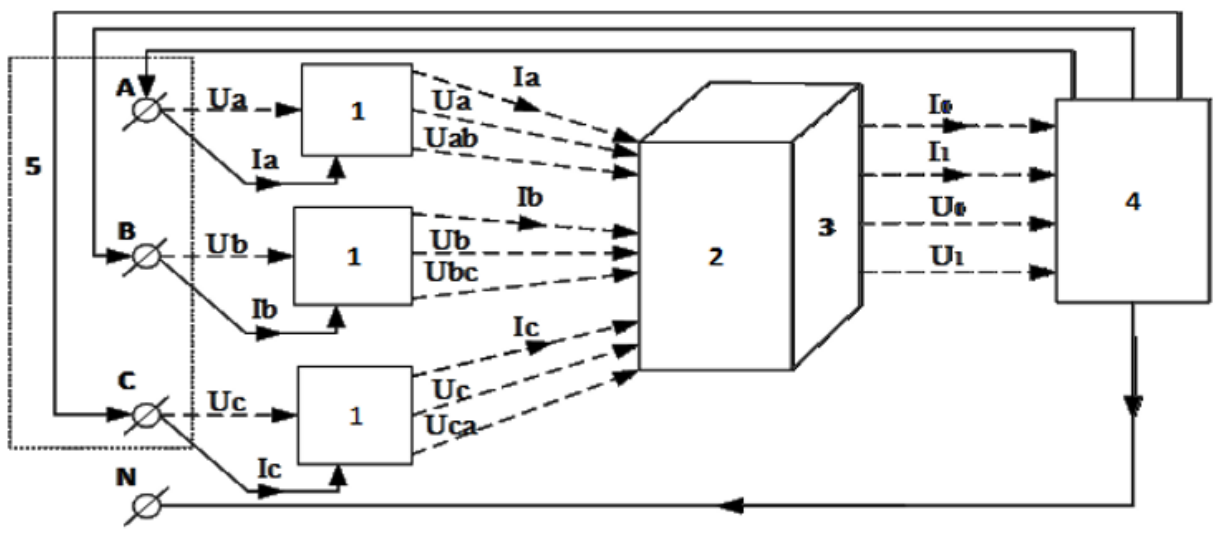

Fig. 1. The "GSG Technology" implementation functional scheme to assess and minimize the unbalancing mode consequences.

1 - Optical current (voltage) transformer. Their use in modern power supply systems is due to the fact that it is quite problematic to integrate analog TC and TV into a single monitoring and control network and the measurement accuracy no longer meets modern standards and requirements, since, according to our country general development the entire power system strategy, all substations should be digital format reconstructed pretty soon. At the same time, such devices advantages are obvious: no resonance, high linearity, no influence of the secondary circuits load, and most importantly - a very wide frequency range and increased safety. The such operation principal converters are based on the Faraday effect use, according to which, during the linearly polarized light propagation located in a magnetic field, the light polarization is plane observed rotation, that is, depending on the beam deflection angle, the electronics calculates the flowing current taking into account all existing errors. The optical sensor, located perpendicular to the current-flow tire, does not contact with it, and the signal is transmitted digitally via the shared bus to the electronics module personal computer -2 . 
Thus, in accordance with IEC [21] the currents and voltages instantaneous values transfer occurs 80 times in the industrial frequency each period in digital form. At the receiving end ( 2 - computer), this information is collected and processed (filtering, digital processing, etc.) - it is supposed to collect 96 points for a period and transmit 2 measurements in one package.

3 - The calculation methods software implementation is based on the methods compilation [7-10]. The "Modulomethod" program algorithm is shown in Figure 2.

The digital data currents and voltages reception in from optical current transformers is supplemented by data on the electrical network parameters entered into the program: the high and low voltage lines full complex resistances and power transformer full complex resistances. In addition, it is possible to integrate data on three-phase symmetrical load into the program (asynchronous motors - if they are available in the power consumption system). The studied power quality indicators and its loss changes time diagrams visualization, in accordance with the calculation algorithm, is carried out on a computer monitor and its hard disk and can be showered at any required time. At the same time, the electrical network operation options are taken into account, both without balancing means, and when a balancing device is included in the any consumers concentration load studied node, as well as on the power transformer buses.

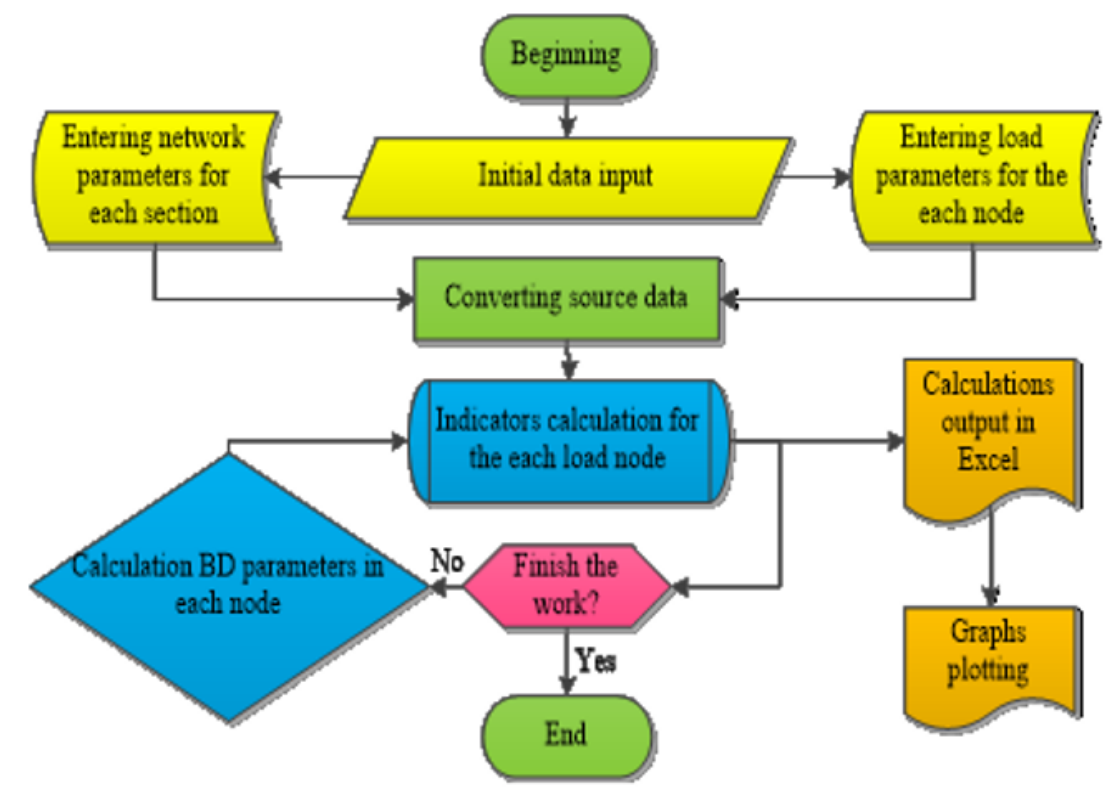

Fig. 2. The implementing calculations algorithm in the software "GSG Technology" using the program "Modulomethod".

4 - A balancing device. The traditional balancing means use is limited by their unregulated parameters constancy. This leads to additional losses in the device itself and significantly increases costs.

Therefore, the most optimal are BD, which change their power as the current unbalance changing level function. The proposed model prototype is an electromagnetic device described in [22]. The following an electromagnetic device model is proposed, which windings are connected according to the counter zigzag scheme, and are located on rigid fixed bases (frames) 7. Movable anchors 5, acting as a magnetic circuit, are fixed rigidly on one side on a spring bundle 2 (Figure 3 ). 
The proposed device operation principle is based on the electromagnetism laws and is as follows. Before the device starts working, the steel cores (armature) 5, due to their own weight, enter the cylindrical windings 3 and 4, connected according to the counter zigzag scheme in each phase, at a $d l_{1}$ distance. At the same time, the all three cores gravity $\left(F_{\text {core }}\right)$ will be balanced by the three sprin tensile force 2: $F_{\text {core }}=F_{\text {spring. }}$. Since the windings connection according to the counter zigzag scheme reduces the windings resistance to zerosequence currents by more than two magnitude orders, the total zero-sequence current is closed in the area between the device connection point and the unbalanced load. When the current passes through the coil, a magnetic field arises in it, which is uniform inside the cylindrical coil and is expressed by the: $B=\frac{\mu_{0} \cdot I \cdot W}{l_{\mathrm{K}}}$. The armature 5 will be affected by the electromagnetic force $\mathrm{F}_{\mathrm{em}}$, which at some point in time is balanced by the spring tensile force: $F_{\text {spring }}=F_{\text {core }}+F_{\text {em }}$. Since the current at each time moment in the three windings each is unchanged, the zero-sequence current also expends energy to increase the flow coupling in the $i d \Psi$ amount, which is half the magnetic field energy. The other half is spent on covering the mechanical work $F_{e m} \cdot d l$ in moving the core inside each phase winding. With an increase in the unbalanced power consumption, the zero sequence current increases, thereby increasing the electromagnetic field energy, cause an increase in the coil inductance. The cores 5 are drawn into each frame 7 and occupy the position $d l_{2}$. In this case, the magnetic field energy in each winding changes by the valued: $d W_{\mathrm{H}}=1 / 2 \cdot i d \Psi$. When the zero sequence current decreases, the magnetic energy decreases, thereby reducing the inductance, i.e., the inductance self-regulates due to the force's equilibrium $F_{\text {spring }}=$ $F_{\text {core }}+F_{\text {em }}$. It should be noted that in this case, the third-party forces energy is not used, and the work is carried out due to the zero-sequence current. It should be noted that in this case, the third-party energy forces is not used, and the work is carried out due to the zero sequence current. That is, as the flow coupling $d \Psi$ increases (decreases) in each winding, the inductance $d L=d \Psi / i$ increases (decreases) depending on the zero-sequence current magnitude. Thus, the cores movement inside the windings each will be determined by each phase zero sequence current magnitude. In other words, each phase inductors regulate their inductance depending on the current magnitude passing through them, therefore, their reactive conductivity $Y_{L}$ is a zero-sequence current function $Y_{L}\left(I_{0}\right)$.

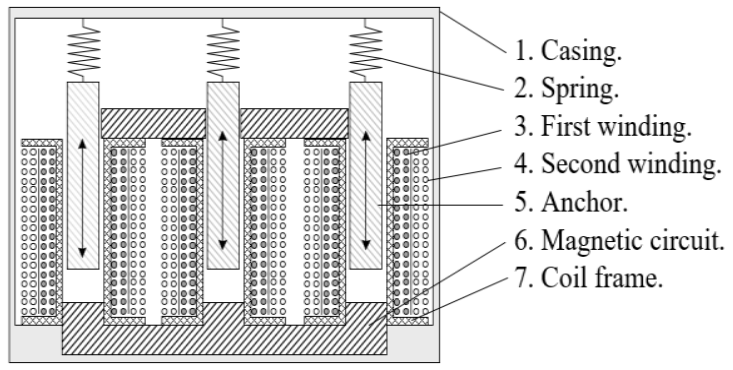

a)

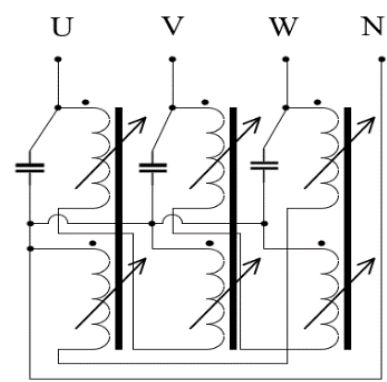

b)

Fig. 3. Electromagnetic balancing device: a) - design, b) - electrical circuit.

This balancing device advantage is that the inductors conductivity, and, consequently, the device power, is self-regulated by the zero-sequence current passing through it and, with a minimum (threshold) unbalancing power consumption level value, can be disconnected from the electrical network by a switching device means. Based on the above, the device parameters are adjusted precisely from the positive and zero sequence currents. 
Based on the methods use described in [8-9], the balancing device parameters can be determined by the expressions:

$$
Y_{B D 1}=Y_{B D 2}=\frac{1}{\left(2 * \frac{U_{1}}{I_{1}}\right) * 7.0714} ; Y_{B D 0}=2 *\left(\frac{3 * I_{0}}{U_{0}}\right)
$$

Were $U_{1}, U_{0}, I_{1}, I_{0}$ - the symmetrizing components of voltages and currents positive and zero sequences, determined, respectively, by expressions (4).

\section{Results and discussion}

\subsection{Unbalancing operation modes investigation}

To test the proposed technology, measurements were used in the current $0.38 \mathrm{kV}$ network made in accordance with the CJSC IRMET Protocol (Electrical energy test report dated 02.27.2020). The tests were carried out by the JSC Irkutsk Electric Grid Company order (665709, Irkutsk region, Bratsk-9, s/b 786). The tests duration is 24 hours, the measurement interval is 1 minute. The measurements were carried out by a certified RESURS UF2M device (factory No. 2337) at the electricity transmission point to consumers on the TMG250-10/0.4 power transformer $0.4 \mathrm{kV}$ tires, which receives electricity via a $10 \mathrm{kV}$ power transmission line made by AC70 wire, $1.259 \mathrm{~km}$ long. The device was installed on an outgoing $0.38 \mathrm{kV}$ line, made with an insulated SIP-2A $3 \times 25+1 \times 35 \mathrm{~mm} 2$ wire with a 0.270 $\mathrm{km}$. length. Utility consumers (individual residential houses) with electric energy threephase input are connected to the line. The electrical network under study has the following parameters.

The $10 \mathrm{kV}$ line positive (negative) sequence total complexes resistances: $Z_{L H 1}=Z_{L H 2}=0,579+j 0,346 \mathrm{Ohms}$. The power transformer positive (negative) and zero sequence total complexes resistances are equal, respectively: $Z_{T 1}=Z_{T 2}=0,0094+j 0,0272$ Ohms, $Z_{T 0}=0,096+j 0,2349$ Ohms. Similarly, for the low voltage lines: $Z_{L L 1}=Z_{L L 2}=0,324+j 0,022358$ Ohms; $Z_{L L 0}=0,3076+j 0,0219$ Ohms.

Power transformer data: idling power $P_{i d}=580 \mathrm{~W}$; short circuit power $P_{\text {sh, } \text {,irc. }}=4200 \mathrm{~W}$; idling current $I_{i d}=1.2 \%$; short circuit voltage $u_{\text {sh.circ. }}=4.7 \%$.

Based on the measurements made and the "Modulomethod" software use, changes time diagrams in currents and voltages in the studied electrical network were obtained (Figure 4). This figure analysis showed the following. As can be seen, the currents in the phases (Fig. 2, a) take different values. At the same time, phase " $\mathrm{A}$ " is the most loaded. The average current value in this phase during the measurement period was $45.28982 \mathrm{~A}$. In the other two phases (B and $\mathrm{C}$ ), the difference in current changes is not so clear, their values are on average 14.91498 and 17.7505 A, respectively. At the same time, the phase and phase-to-phase voltages averages values for the measurement period (Fig. 4) are, respectively: $U_{A}=232.6257 \mathrm{~V}, U_{B}=235.7242 \mathrm{~V}, U_{C}=234.1277 \mathrm{~V}, U_{A B}=404.6016 \mathrm{~V}$, $U_{B C}=407.0769 \mathrm{~V}, U_{C A}=405.0429 \mathrm{~V}$. As can be seen, three-phase power supply voltage system unbalance is not as clearly represented as it is typical for currents in phases. 


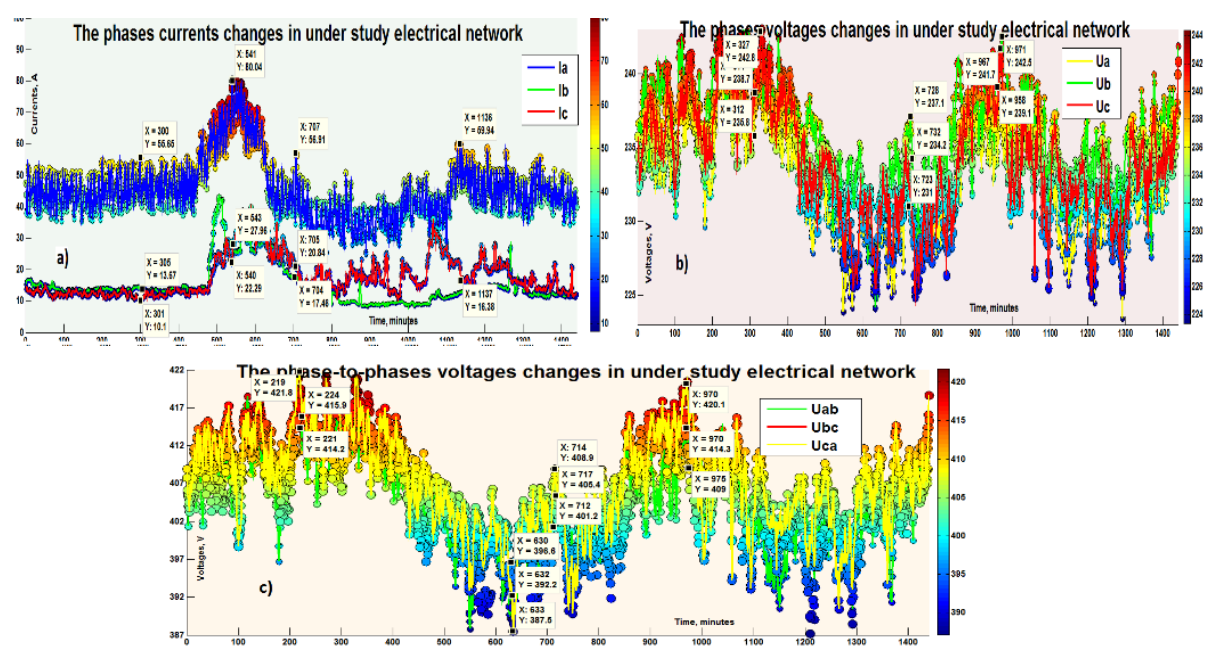

Fig. 4. The changes time diagrams in phase currents, phase and phase-to-phase voltages in the line under study.

To analyze the indicators determined by the expressions (1), (2) and (3) and the "GSG Technology" use presented in Figure 1, their calculation was performed and, based on the MATLAB graphics editor technologies use, the changes time diagrams in these indicators were constructed (Fig. 5-10). These diagrams clearly show the balancing device effect, which parameters, in accordance with Fig. 1, automatically change in accordance with the change in the unbalanced load power. The change in the negative sequence voltage unbalance coefficient is not considered in this article, since its value in the networks feeding the utility consumers load is minimal, and the balancing effect according to this coefficient is either weakly expressed or the opposite, is absent. It is since the balancing device parameters are calculated from the positive and zero sequences currents and voltages unbalancing indicators only.

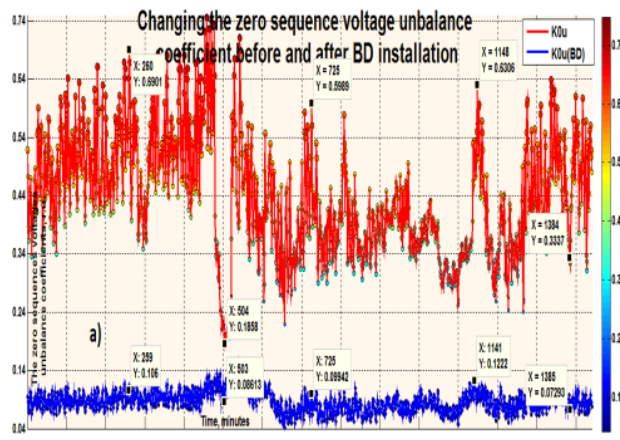

a)

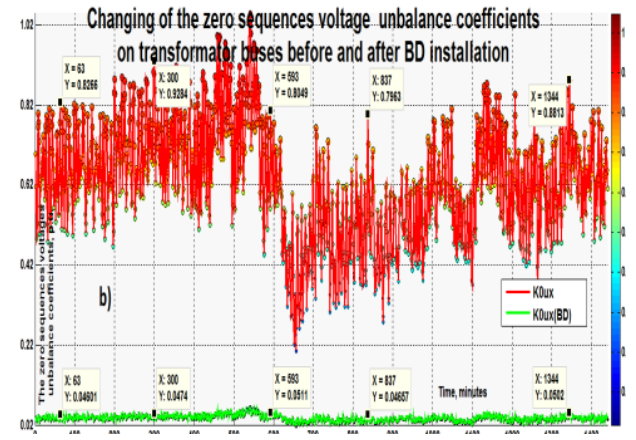

b)

Fig. 5. Changing time diagrams the zero-sequence voltage unbalance coefficient in the load node (a) and on the power transformer buses (b).

Figure 5 shows changes diagrams in the zero-sequence voltage unbalance coefficient in the power take-off node (at the $0.27 \mathrm{~km}$ distance from the power transformer $0.4 \mathrm{kV}$ tires), as well as directly on the $0.4 \mathrm{kV}$ tires. The changes analysis in these indicators showed the following. The power quality level according to the $K_{O U}$ indicator meets the Standards requirements [1-3]. So, the average for the measurement period, this indicator value in the load node (Fig. 5, a) was $0.44 \%$, on the transformer tires (Fig. 5, b) $-0.63 \%$. The balancing 
device effect when it is connected is manifested in a significant decrease in $K_{0 U}$ - in the node to $0.087 \%$ (the balancing effect was $80.3 \%$ ), on the tires - to $0.0377 \%$ (the balancing effect is $94 \%$ ).

The power quality according on the voltage steady-state deviation does not meet the Standard's requirements. The $\delta U$ indicator value at the electric energy transmission point (on transformer buses) should not exceed $10 \%$. At the same time, at the general connection point consumers to the electrical network, this indicator value in the $95 \%$ measurement time interval should not exceed $\pm 5 \%$. Figure 6 shows changes time diagrams in steadystate voltage deviations in the load node (the common connection point) when the absence and balancing device inclusion.

The Figure 6 analysis showed the following. In the BD absence, the average voltage deviation $(\delta U)$ value during the measurement period was $6.73 \%$, and the output beyond the established norm (5\%) was observed in the $93.2 \%$ measurement interval time. Thus, the power quality according to the established voltage deviation does not meet the Standard's requirements. When $\mathrm{BD}$ was turned on in the load node, the average $\delta U$ value decreased to $0.252 \%$ (balancing effect was $96.3 \%$ ).

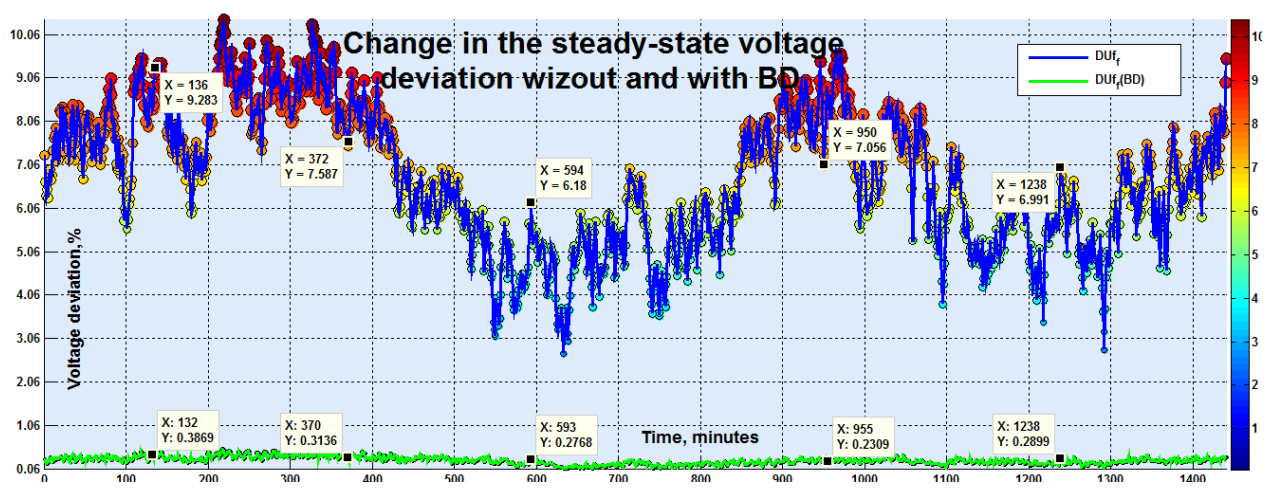

Fig. 6. Changes time diagrams the phase-to-phase and phase steady-state voltage deviations in the absence and BD inclusion.

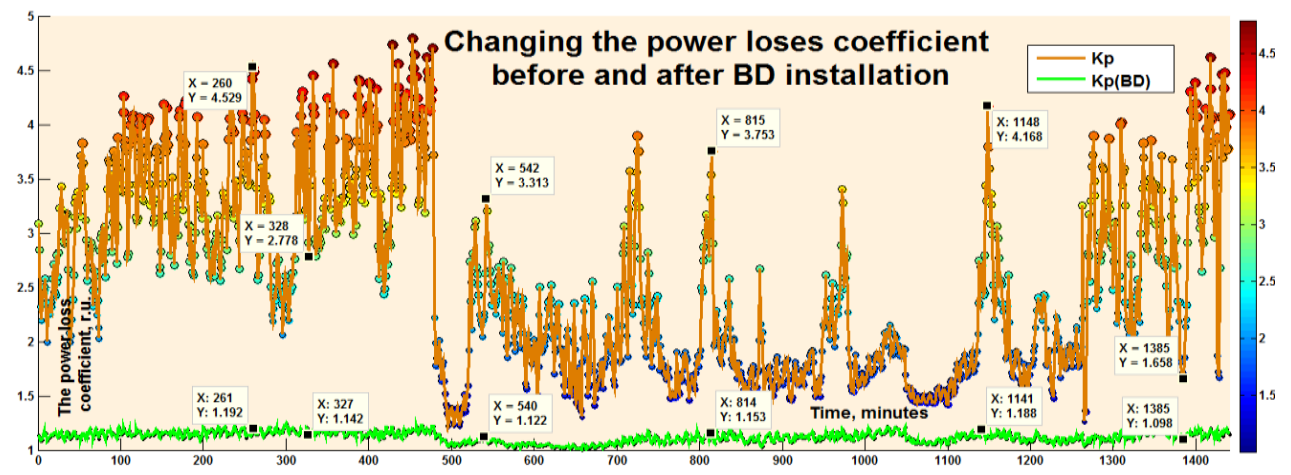

Fig. 7. The power loss coefficient time diagrams changing in the studied electrical network when the BD absence and its inclusion. 


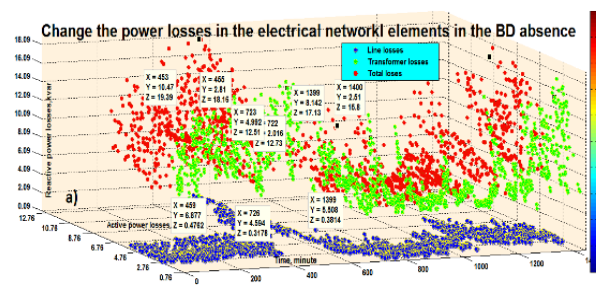

a)

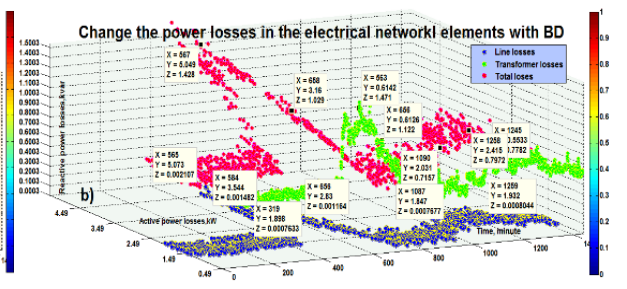

b)

Fig. 8. The BD symmetrizing effect visualization when power losses change in the electrical network elements: a) BD absence; b) BD is turned in the load node.

Let's consider how the power loss coefficient changes, which characterizes the increase in the unbalanced mode losses.

Figure 7 shows the change time diagrams in the power loss coefficient when the absence and BD inclusion in the load node.

Based on the Figure 7 analysis, it was found that in the BD absence, the power loss coefficient when it changes during the measurement time interval takes different values from the minimum (1.22) to the maximum (4.79). Its average measurement period value was 2.54. This means that the electrical energy losses caused by unbalancing power consumption are more than 2.5 times higher than those losses that could be caused by the only one positive sequence currents flow, that is, losses in a conditionally symmetric (balanced) mode.

When the balancing device is turned on, a significant balancing effect is observed in the load node. The average loss coefficient value during the measurement period was 1.12. That is, the balancing effect is $56 \%$.

Let's analyze how the real electrical energy losses in the network under study change before the BD is turned on and integrated into the load node. Figure 8 shows the power losses change characteristics in the electrical network elements before and after BD switching on. As can be seen from Figure 8, in the BD absence in the $0.38 \mathrm{kV}$ network under study, the active losses in the line during the measurement interval change every minute from 1.3 to $13.2 \mathrm{~kW}$. The average these losses value during the measurement period is $4.377951 \mathrm{~kW}$ per minute. The line reactive power losses, varying from 0.09 to $0.9 \mathrm{kvar}$, averaged $0.3 \mathrm{kvar}$ per minute. The line average full power losses are $4.388413 \mathrm{kVA}$. In a power transformer, active losses averaged $1.531783 \mathrm{~kW}$ over the period, reactive power $9.771732 \mathrm{kvar}$, full power $-9.89115 \mathrm{kVA}$. When the balancing device was switched on, the line active, reactive and full power losses were, respectively: $1.924977 \mathrm{~kW}, 0.00079 \mathrm{kvar}$ and $1.924977 \mathrm{kVA}$. Thus, the line active power loss balancing effect amounted to almost $66 \%$, reactive power loss - almost $100 \%$, and more than $56 \%$ of the total power loss. In the power transformer, the BD inclusion led to a reduction in active power losses by $65 \%$, reactive power by $92.2 \%$, and full power by $90.5 \%$.

The change in total full power losses is shown in Figure 9. 


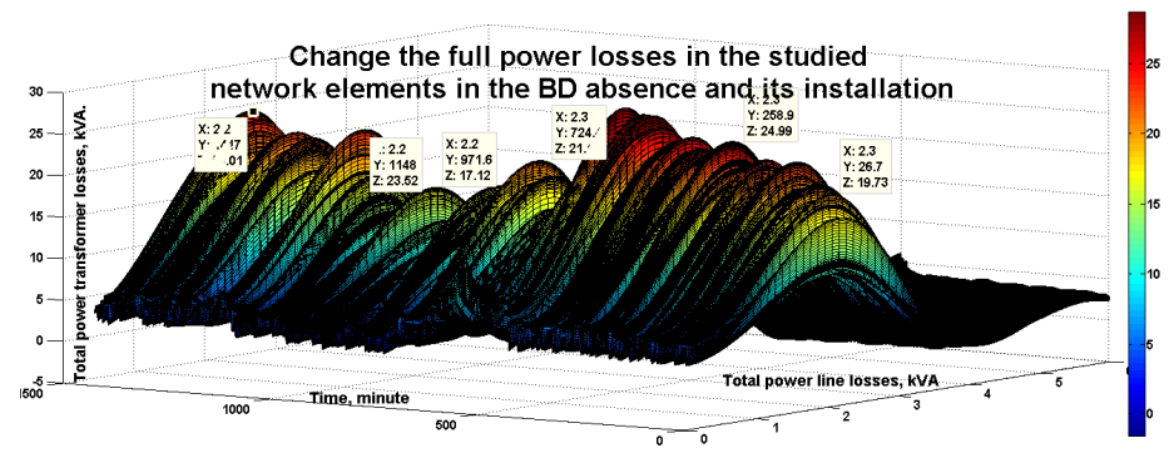

Fig. 9. The symmetrizing efficiency when changing the total full power in the electrical network elements.

The Figure 9 analysis showed the following. In the balancing device absence in the studied network, the average full power total loss per minute is $14.27956 \mathrm{kVA}$. The active power accounts share $5.909734 \mathrm{~kW}$ these losses, the reactive power share $-10.07459 \mathrm{kvar}$ per minute. The balancing device inclusion leads to the average full total losses decrease on the $2.863912 \mathrm{kVA}$ value. There are $2.46311 \mathrm{~kW}$ is the active power accounts of these full loses and $0.765389 \mathrm{kvar}$ is for the reactive power. Thus, judging by the active power losses, the balancing device allows use us to save $3.53423 \mathrm{~kW}$ every minute. Under the investigated electrical network functioning condition without disconnections for one year, the electric energy savings will amount to $1857591.288 \mathrm{kWh}$. With the electric energy cost in the rural Irkutsk region areas equal to 0.861 rubles per $1 \mathrm{kWh}$ [22], the saved losses cost will amount to $1599.386,098968$ rubles. Considering that the creating cost technology in question is about $25-30 \%$ [16], the real using "GSG Technology" annual economic effect for the studied electrical network may amount to 479815.83 rubles. or $6771.32 \$$. It should be noted that in this case, the improving power quality economic effect is not taken into account.

In addition, the balancing device costs described in [8] imply BD with the unregulated parameters use when its installed capacity is constantly used. In the regulated devise parameters use case described above, the BD power used and losses in it will be determined only by the current unbalance level, so the specified effect can be increased.

\subsection{The studied indicators predictive evaluation mechanism}

Common forecasting methods include statistical methods and Data Mining methods. Statistical methods include probability theory and mathematical statistics, regression analysis, maximum likelihood, time series extrapolation methods. Probability distribution laws are used to estimate many randomly varying parameters, among which we distinguish normal, gamma, Weibull, Pearson type III, three-parameter power law, etc. [24, 25]. One of the constructing conditions the probability distribution law is an autocorrelation relationship absence between the parameter under study values. To identify the relationship between the sample values or to refute this hypothesis, an autocorrelation coefficient calculated by the formula is used:

$$
R_{\tau}=\frac{\sum_{i=1}^{n-\tau}\left(x_{i}-\bar{x}_{i}\right) \cdot\left(x_{i+\tau}-\bar{x}_{i+\tau}\right)}{\sigma_{i} \sigma_{i+\tau}(n-\tau-1)}
$$

Where $n$ - is the sample size; $\tau$ - the shift order, varying from 0 to $m$ ( $\tau=0,1,2, \ldots$, $m) ; x_{i}$ - the series values from $x_{1}$ to $x_{n-\tau}$; and $\sigma_{i}$ - the average value and standard for the 
sample part from 1 to $n-\tau ; x_{i+\tau}$ - the series values from $x_{1+\tau}$ to $x_{n} ; \bar{x}_{i+\tau}$ and $\sigma_{i+\tau}-$ the average value and standard for a sample size from $1+\tau$ to $n$.

The studied series parameters autocorrelation coefficients calculation showed that in most cases the intra-row connection is strong and significant $\left(\mathrm{R}_{1}>0.8\right)$. In this regard, the probabilistic laws construction is not possible. At the same time, autoregressive dependencies can be used to predict the studied indicator values in the short term, according to which the subsequent series values depends on the previous one. In other words, if the parameter value in question increased in the previous period (month, year), it will increase in the next, if there is a direct relationship between the values. In the case when the relationship is reversed (the autocorrelation coefficient is a negative value), the future value will decrease with an increase in the previous one.

The Fischer criterion was used to check the autoregressive model's quality shown in Table 1. The equations coefficients significance is estimated using the Student's criterion.

Table 1. The autoregressive models studied indicators constructed by minutes.

\begin{tabular}{|c|c|c|}
\hline Indicator & Equation & Determination coefficient \\
\hline $\begin{array}{c}\text { The zero-sequence voltage } \\
\text { unbalance coefficient }\end{array}$ & $y_{t}=0,88 y_{t-1}+0,054$ & 0,77 \\
\hline Power loss factor & $y_{t}=0,94 y_{t-1}+0,15$ & 0,86 \\
\hline Total losses & $y_{t}=0,90 y_{t-1}+1,40$ & 0,81 \\
\hline \multicolumn{2}{|c|}{ with a balancing device } \\
\hline $\begin{array}{c}\text { The zero-sequence voltage } \\
\text { unbalance coefficient }\end{array}$ & $y_{t}=0,83 y_{t-1}+0,015$ & 0,68 \\
\hline Power loss factor & $y_{t}=0,90 y_{t-1}+0,12$ & 0,81 \\
\hline Total losses & $y_{t}=0,97 y_{t-1}+0,081$ & 0,94 \\
\hline
\end{tabular}

The obtained equations can be used to predict the studied indicators in the short term.

Among the Data Mining methods, neural networks, decision trees, a genetic algorithm, the support vector method, and others are distinguished. We have adopted the neural network method as the basis, as the most commonly used in the preventive events assessment in electric power systems, the MATLAB modeling environment has been chosen as a creating means a neural network, since it is easy to use and has a high operation speed. In addition, there are a large methodological number materials on its use, which is very potential users valuable [26-28]. At the first stage, using autocorrelation coefficients, the analyzed indicators intra-row relationship was estimated, according to which there is a strong significant autocorrelation relationship in all variables, which makes it possible to use autoregressive forecasting models. To create a neural network, one of the design and neural networks training main MATLAB tools, Neural Time Series, was used.

A nonlinear regression is used as a tool (Fig. 10), which implements the simplest direct propagation network and allows predicting the effective feature value taking into account previous values with a different shift d. Let's consider the power loss coefficient $K p$, like one of the three analyzed indicators.

To check and test data from 1440 this indicator values (the value corresponding to the minute measurement time interval), a 80:10:10 ratio percent was randomly selected, corresponding to the separation: network training - 80\% (1152 target time intervals), approval - 10\% (144 intervals) and testing - 10\% (144 intervals) (Fig. 11). 


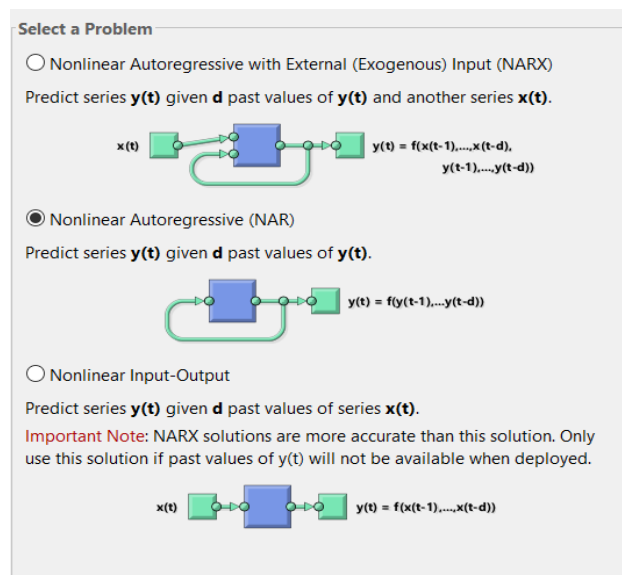

Fig. 10. Nonlinear autoregressive neural network model.

The creation and network training are carried out in an open form cycle, since training with an open loop (one-step prediction) is more efficient than with a closed loop (multistage prediction), and it allows us to provide the network with the correct feedback inputs, even when we train it to get the correct feedback outputs. After training, the network can be transformed into a closed loop form or into any other form required by the application. In this regard, the following architecture was adopted by random iteration: the hidden neurons number is 70 , the delays number is 2 (Fig. 12). When working with the created neural network architecture (Fig. 12), its indicators were obtained (Fig. 13, 14), characterizing the using this model possibility to carry out the change's states preventive assessment in additional energy losses due to phase current unbalance.

8 Randomly divide up the 1440 target timesteps:
Training:
$\begin{aligned} & \text { Validation: } \\ & \text { Testing: }\end{aligned}$
$\begin{array}{ll}10 \% & 1152 \text { target timesteps } \\ & 144 \text { target timesteps } \\ \end{array}$

Fig. 11. Validation and Test Data.

The reference vectors values are laid out in the dotted line form, these corresponding parameters individual values are indicated by the "circle" type markers, the line shows all the studied model values correlation. The overall $\mathrm{R}$ score exceeds 0.8 , which indicates a sufficiently high accuracy and constructed model adequacy.

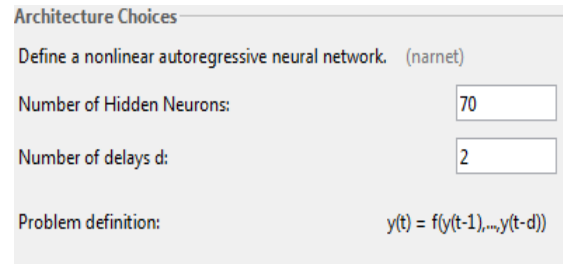

a)

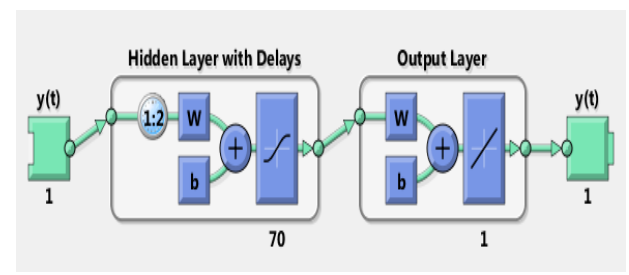

b)

Fig. 12. Building a neural network architecture: a) the neurons and delays number selection; b) the neural network configuration. 


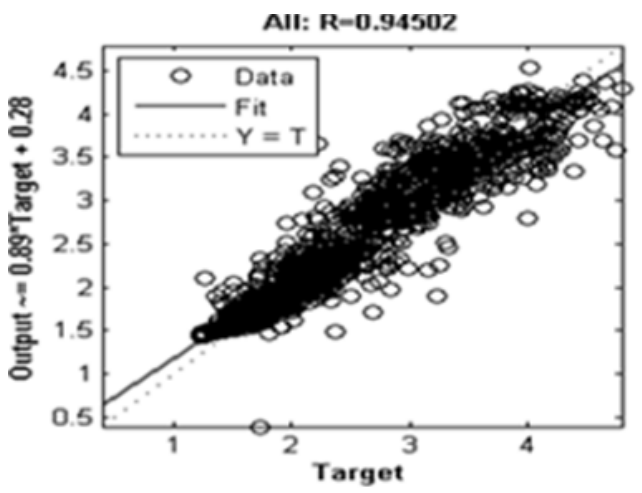

Fig. 13. Diagram of the neural network data general distribution.

Figure 14 shows the neural network training result, according to which the error during training has an insignificant value with some values exception "falling out" from the time series.

A selective retrospective the studied indicators forecast using a neural network showed that the actual values deviation from the forecast does not exceed $3 \%$.

In addition, the loss coefficient retrospective prediction results comparison for the autoregressive equation and the neural network showed that the forecasts average deviation from the actual values is $1.9 \%$, and for the neural network $-2.6 \%$ (Fig. 15). Thus, it can be concluded that linear autoregression describes the original series more accurately than the nonlinear one.
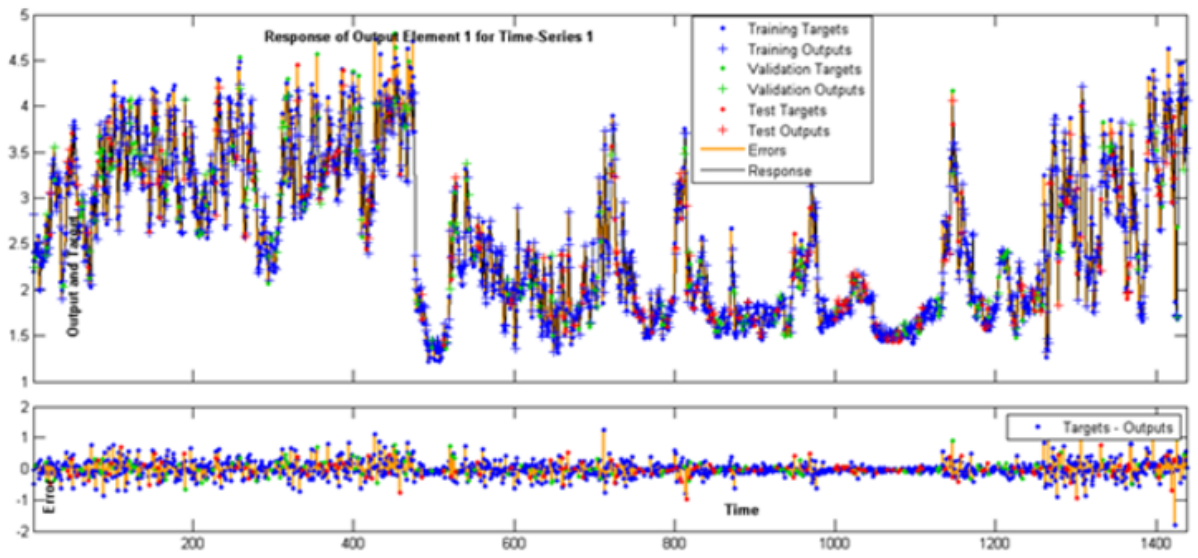

Fig. 14. Time series response in neural network training.

Retrospective forecast, obtained by autoregressive equations and neural networks showed that the actual values deviation from the forecast has a satisfactory value and does not exceed $10 \%$ (Table 2 ). It should be noted that the neural network gives a more accurate forecast. However, training and testing the network using various input indicators requires more time than calculating the autoregressive model parameters.

The graph comparing the initial data and the loss coefficient retrospective prediction data without a balancing device using the autoregressive equation and a neural network (Fig. 15) shows that the predicted values have practically no deviations from the actual ones. 
Table 2. The average the power quality indicators forecast values deviation from the actual data in absolute value, $\%$.

\begin{tabular}{|c|c|c|}
\hline \multirow{2}{*}{ Indicator } & \multicolumn{2}{|c|}{$\begin{array}{l}\text { The forecast value average deviation from the actual } \\
\text { value in absolute value, } \%\end{array}$} \\
\hline & $\begin{array}{l}\text { according to the } \\
\text { autoregressive model }\end{array}$ & by neural network \\
\hline \multicolumn{3}{|c|}{ without a balancing device } \\
\hline $\begin{array}{c}\text { The zero-sequence voltage } \\
\text { unbalance coefficient }\end{array}$ & 7,96 & 7,71 \\
\hline Power loss factor & 7,56 & 7,45 \\
\hline Total losses & 9,67 & 9,44 \\
\hline \multicolumn{3}{|c|}{ with a balancing device } \\
\hline $\begin{array}{c}\text { The zero-sequence voltage } \\
\text { unbalance coefficient }\end{array}$ & 9,01 & 9,01 \\
\hline Power loss factor & 1,43 & 1,41 \\
\hline Total losses & 6,31 & 6,17 \\
\hline
\end{tabular}

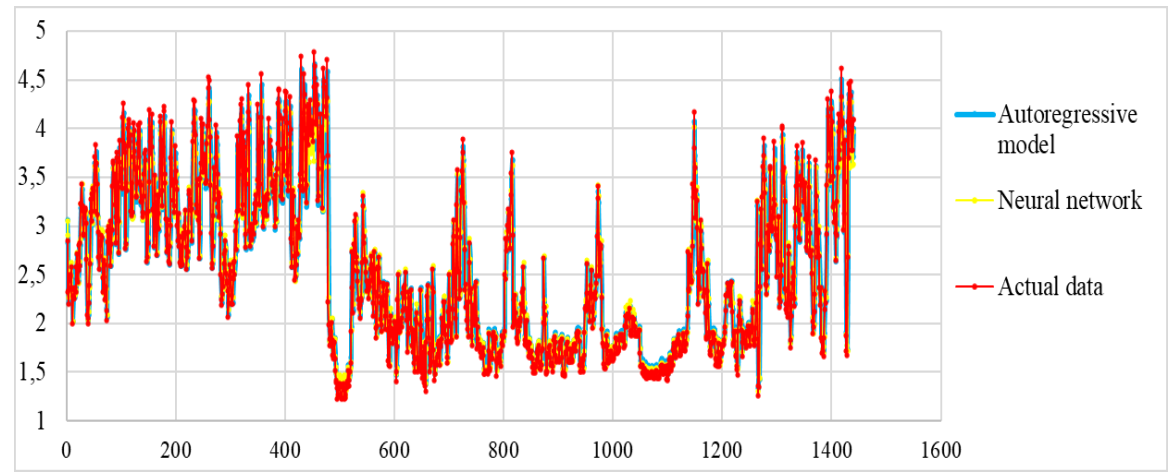

Fig. 15. Actual values and a retrospective forecast result based on an autoregressive model and a neural network.

\section{Conclusions}

Based on the conducted research, the following was established

- $0.38 \mathrm{kV}$ voltage rural electric networks are characterized by the phase currents and voltages unbalance. At the same time, changes in the currents and voltages unbalance are always different on different the three-phase electrical network sections, which is due to the unbalancing power consumption probabilistic nature.

- A digital technology based on the measuring, calculation and decreas of the unbalancing indicators, characterizing the power quality and its loss minimization is proposed to evaluate the $0.38 \mathrm{kV}$ electrical networks unbalancing modes operation.

- Developed: calculation software and a balancing device to minimize the power quality indicators and its losses caused by the currents and voltages unbalance.

- The proposed technology approbation was carried out on the currents and voltages measurements basis in the $0.38 \mathrm{kV}$ electrical network. At the same time, the power quality and its additional losses analysis showed that the using efficiency a balancing device to reduce the power quality established indicators was more than $90 \%$, and to reduce energy losses - close to $60 \%$. The annual reducing energy losses economic effect for the studied electrical network amounted to 479816 rubles. 
- Comparison of the studied indicators preventive assessment methods allowed to establish that the linear autoregression methods use is the most effective for the unbalancing regimes evaluation creating forecasts and minimizing them.

\section{Reference}

1. GOST 32144-2013 INTERSTATE STANDARD. Electric energy. Electromagnetic compatibility of technical equipment. Power quality limits in the public power supply systems Moscow. Standartinform (2014)

2. IEEE 1159-2019 - IEEE Recommended Practice for Monitoring Electric Power Quality (2019)

3. EN 50160:2010/A3:2019. Voltage characteristics of electricity supplied by public electricity networks (2019)

4. I.V. Naumov, N.V. Savina, I. V. Yamshchikova, M. V. Shevchenko, IPASJ International Journal of Electrical Engineering (IIJEE), 5(5) (2017)

5. I. V. Naumov, International Science and Technology Conference "EastConf" INSPEC. Accession Number: 18724557 1-6. (2019)

6. I.V. Naumov, M.V. Shevchenko, P.P. Kozhushko, Journal Trends in the development of science and education, 30(2), 24-27 (2017)

7. F. D. Kosoukhov, The current and voltage unbalance indicators calculation and analysis methods in rural distribution networks, 42 (1984)

8. I. V. Naumov, Methods and technical means the currents unbalance and electric energy losses reducing in rural distribution 0.38 kV networks, 277 (1989)

9. I. Naumov, Reducing losses and improving the quality of electric energy in rural distribution networks of $0.38 \mathrm{kV}$ with the help of symmetric devices, 387 (2002)

10. I. Naumov, E. Belousova, Vestnik Krasgau, 1, 99-107 (2017)

11. A. S. Lukovenko, V. V. Kukartsev, V. S. Tynchenko, A. S. Mikhalev, V. A. Kukartsev, K. A. Bashmur, Journal of Physics: Conference Series. International Scientific Conference "Conference on Applied Physics, Information Technologies and Engineering - APITECH-2019", 55086 (2019)

12. Y. Xie, L. Wang, P. Li, T. Li, X. Su, Journal of Physics: Conference Series, 1549(5), 052053 (2020)

13. G. Bao, S. Ke, Energies, 12(15), 2842 (2019)

14. I. V. Naumov, S. V. Podyachikh, IOP Conf. Series: Earth and Environmental Science, 808, 012006 (2021)

15. I. Naumov, S. Podyachikh, D. Ivanov, A. Tretyakov, A. Bastron, International Scientific Forum on Sustainable Development and Innovation (WFSDI 2021) E3S Web of Conferences, 295, 02005 (2021)

16. I. V. Naumov, S. V. Podyachikh, D. A. Ivanov, SAHD 2021. SHS Web of Conferences 101, $02012(2021)$

17. D. E. Dulepov, T. E. Kondranenkova, Bulletin of the NGIEI, 4(71), 28-35 (2017)

18. A. V. Vinogradov, A. V. Vinogradova, I. O. Golikov, V. E. Bolshev, International Journal of Emerging Electric Power Systems, 20(3), 3 (2019)

19. G. V. Lukina, I. V. Naumov, A. A. Lukin et al., RF Patent no. 26699 RF, MPK7 H 02 J $3 / 26(2003)$ 
20. I. V. Naumov, D. A. Ivanov, S. V. Podyachikh, et al., RF Patent no. 2490768 RF, MPK6 H 02 J 3/26 (2013)

21. GOST R 54835-2011/IEC/TR 61850-1:2003. Communication networks and systems in substations - Part 1: Introduction and overview (IDT), Moscow. Standartinform, 2012. The introduction date is 2012-09-01 (2012)

22. A.K. Shidlovskij, V.G. Kuznetsov, N.N. Kaplychnyj, et al., SU Patent no. 1206881 SU, MПК Н 02 J 3/26, (1986)

23. Tariffs for Electric Energy for the Population of the Irkutsk Region and Consumers Equated to the Population. https://sbyt.irkutskenergo.ru/

24. S.V. Volkov, L.M. Rubakov, Energy problems, 1, 84-89 (2004)

25. M.A. Undin, E.V. Rud, D.N. Golovakha, Collection of articles of the scientific and practical conference: Advanced achievements in the application of automation, robotics and electrical technologies in the agro-industrial complex, 190-195 (2019)

26. L.P. Bilgaeva, K.G. Vlasov, He Study of Models of Neural Network Forecasting in Matlab, 1(7), 11-19 (2017)

27. J. MacLennan, Z. Tang, B. Crivat, Data Mining with Microsoft SQL Server 2008, 675 (2009)

28. Oracle Cryslat Ball, http://www.hyperion.ru/ 